\title{
Cardiorespiratory fitness in breast cancer survivors
}

\author{
David Burnett ${ }^{*}$, Patricia Kluding, Charles Porter, Carol Fabian and Jennifer Klemp
}

\begin{abstract}
Maximal oxygen uptake $\left(\mathrm{VO}_{2 \max }\right)$ has been used to assess risk for all-cause mortality and cardiovascular disease $(\mathrm{CVD})$, and low $\mathrm{VO}_{2 \max }$ has recently been associated with increased mortality from breast cancer. The purpose of this study was to determine the proportion of breast cancer survivors with 2 or more risk factors for CVD exhibiting a low $\mathrm{VO}_{2 \max }$ and to determine whether sub-maximal endpoints which could be applied more readily to intervention research would correlate with the maximal treadmill test. We performed a single $\mathrm{VO}_{2 \text { max }}$ test on a treadmill with 30 breast cancer survivors age 30-60 (mean age $50.5 \pm 5.6$ years) who had 2 or more cardiac risk factors for CVD not related to treatment and who had received systemic therapy and or left chest radiation. Submaximal $\mathrm{VO}_{2}$ endpoints were assessed during the $\mathrm{VO}_{2 \max }$ treadmill test and on an Arc trainer. Resting left ventricular ejection fraction (LVEF) was also assessed by echocardiogram (ECHO) or multi-gated acquisition scan (MUGA). A majority (23/30) of women had a $\mathrm{VO}_{2 \max }$ below the 20th percentile based on their predicted normal values. The group mean resting LVEF was $60.5 \pm 5.0 \%$. Submaximal $\mathrm{VO}_{2}$ measures were strongly correlated with the maximal test including; 1$) 85 \%$ age predicted maximum heart rate $\mathrm{VO}_{2}$ on treadmill, $\left.(r=.89 ; p<0.001), 2\right)$ treadmill $\mathrm{VO}_{2}$ at anaerobic threshold (AT), $(r=.83 ; p<0.001)$, and 3) $\mathrm{Arc}_{\mathrm{VO}}$ at $\mathrm{AT},(r=.80 ; p<0.001)$. Breast cancer survivors with 2 or more CVD risk factors but normal LVEF had a low cardiorespiratory fitness level compared to normative values in the healthy population placing them at increased risk for breast cancer and cardiovascular mortality. Submaximal $\mathrm{VO}_{2}$ exercise testing endpoints showed a strong correlation with the $\mathrm{VO}_{2 \max }$ test in breast cancer survivors and is a good candidate for testing interventions to improve cardiorespiratory fitness.
\end{abstract}

Keywords: Anaerobic threshold, Weight loss, Cardiovascular disease, Chemotherapy, Cardiorespiratory fitness, Exercise testing

\section{Introduction}

More than 290,000 women were estimated to be diagnosed with breast cancer in 2012, and the number of survivors has risen beyond 2.5 million (ACS 2012). There is an excess of cardiovascular deaths in breast cancer survivors (Eloranta et al. 2012). Likely due to physical inactivity and weight gain as well as side effects of treatment (Ewer et al. 2011; Jones et al. 2007a). Low cardiorespiratory fitness usually measured by oxygen uptake at maximal or peak exercise (VO2 max or peak) is inversely correlated with cardiovascular and all cause mortality, and breast cancer deaths (Blair et al. 1996; Blair et al. 1989; Peel et al. 2009). Women with low

\footnotetext{
* Correspondence: dburnett@kumc.edu

Breast Cancer Survivorship Center, Mail stop 5015, University of Kansas Medical Center, 3903 Rainbow Boulevard, Kansas City, KS 66103-9815, USA
}

cardiorespiratory fitness (below $\mathrm{VO}_{2 \max }$ of 8 METS ( $28 \mathrm{~mL} \mathrm{~kg}^{-1} \cdot \mathrm{min}^{-1} \mathrm{VO}_{2}$ or below) had a nearly 3 fold increase in breast cancer deaths compared to those who reached a level above 8 METS (Peel et al. 2009). A recent study indicated breast cancer survivors had a $22 \%$ lower $\mathrm{VO}_{2 \max }$ compared to their age matched healthy, sedentary non-cancer peers (Jones et al. 2012).

While a maximal exercise test is the gold standard for assessing cardiorespiratory fitness, a maximal cardiorespiratory exercise test requires participants to push themselves to volitional exhaustion and peak heart rate (HR) (ACSM 2009). In contrast, submaximal testing allows for a measure of sustainable cardiorespiratory exercise capacity (Wasserman et al. 1999), without maximizing heart rate. In addition, submaximal exercise testing is 
less expensive than maximal testing in that it does not require physician presence during the test.

Submaximal exercise testing has been used to provide a measure of cardiorespiratory fitness, with outcomes based on anaerobic threshold in patients with a history of congestive heart failure (Kemps et al. 2008), stroke (Eng et al. 2004), cancer (Carlson et al. 2006), and athletic populations (Bernardi et al. 2010). Furthermore, submaximal testing is more feasible for use in deconditioned women who may discontinue a maximal test early because of fatigue - a complaint in $50-80 \%$ of breast cancer survivors who have undergone systemic therapy (Andrykowski et al. 1998; Gitt et al. 2002).

A recent meta-analysis indicates a need for a standardized and objective measure of fitness which can be readily utilized in the breast cancer survivorship population (McNeely et al. 2006). Accordingly, we investigated the association between the gold standard measure of cardiorespiratory fitness $\left(\mathrm{VO}_{2 \max }\right)$ with different submaximal testing endpoints in breast cancer survivors. We hypothesized the $\mathrm{VO}_{2}$ at submaximal endpoints would be highly correlated with $\mathrm{VO}_{2 \max }$ in breast cancer survivors and they would have a lower $\mathrm{VO}_{2 \max }$ than age and gender matched normative values. Important clinical implications can arise from evaluating cardiorespiratory fitness in breast cancer survivors and targeting those in the greatest need of preventive measures.

\section{Materials and methods \\ Study participants}

This was a cross-sectional study using a within-subject design to investigate the cardiorespiratory fitness and associations between submaximal and maximal cardiorespiratory tests in breast cancer survivors. A total of 30 breast cancer survivors were enrolled in the study. Recruitment was performed by the Breast Cancer Survivorship Navigator/Coordinator from long-term survivorship clinics at the University of Kansas Breast Cancer Survivorship Center (BCSC). Inclusion criteria were as follows: previously diagnosed with stage I-IIIa breast cancer; ages 30-60; no evidence of metastatic disease; at least 3-months from completing initial chemotherapy and/or radiation therapy; and within 10 years of initial diagnosis. Subjects had to also have 2 of the following cardiac risk factors: body mass index $(\mathrm{BMI}) \geq$ $25 \mathrm{kgm}^{-2}$, hypertension (systolic blood pressure $\geq$ $140 \mathrm{~mm} \mathrm{Hg}$ and/or diastolic blood pressure $\geq 90 \mathrm{~mm}$ Hg),(ACSM 2009) elevated low density lipoprotein (LDL) (fasting serum level $\geq 130 \mathrm{mg}^{-1} \mathrm{dL}^{-1}$ ) (NCEP 2002), positive family history of myocardial infarction, coronary revascularization, or sudden death in mother, father, or first-degree relative, sedentary lifestyle (not currently participating in habitual exercise of moderate to vigorous intensity for two or fewer sessions per week), smoker (currently smoking or quit smoking within the last six months); and 1 treatment-related risk factor: chemotherapy +/- left breast radiation; and available results of a resting ECHO or MUGA prior to receiving initial chemotherapy. Objectives were to analyze $\mathrm{VO}_{2 \max }$ and submaximal endpoints in breast cancer survivors who had been treated with adjuvant therapy. The submaximal tests utilized a treadmill and an Arc Trainer (Cybex Arc Trainer 750AT). An arc Trainer was used instead of a treadmill in cases where minimization of vertical stress was needed such as women with knee and back problems. Arc Trainers use a low-impact mechanical design with production of lower vertical forces and joint loading compared to walking on a treadmill ( $\mathrm{Lu}$ et al. 2007; Turner et al. 2010).

The study was approved by the University of Kansas Medical Center Human Subjects Committee (HSC \#12251) and written informed consent was obtained from all participants at an orientation meeting prior to conducting any study testing or activities.

\section{Outcomes}

All study procedures were conducted during three testing sessions within a two week time frame at the Clinical Translational Science Unit (CTSU) on the University of Kansas Medical Center campus and the cardiovascular lab located at the University of Kansas Hospital. Participants were instructed to refrain from exercise 48 hours prior to maximal and submaximal cardiorespiratory testing. In addition, all participants were asked not to consume any food, caffeine, alcohol, and tobacco products three hours prior to exercise tests. The exercise testing sessions were between 2 and 14 days apart and at the same time of the day. All cardiorespiratory tests followed the American College of Sports Medicine (ACSM) guidelines for procedures during graded exercise testing (ACSM 2009). In addition, the exercise testing sessions were at least 48 hours, but no more than 14 days, from the resting $\mathrm{ECHO} /$ MUGA. Data on demographic information and health history were obtained (Table 1).

\section{Maximal treadmill test}

Cardiorespiratory fitness was assessed using a modified Balke protocol (Balke 1959; Blair et al. 1989). Tests were conducted by qualified personnel including an exercise physiologist, nurse, and CTSU medical monitor. An integrated metabolic measurement system (Parvo Medics TrueOne 2400) was used for measurement of maximal oxygen consumption interfaced with an ECG system (Schiller AT-10 Cardiograph). The metabolic cart was set to produce a 15 -second average of the data collected during gas analyses for all tests. Equipment was calibrated per manufacturers recommendation prior to testing. Participants were fitted and acclimated to a 
Table 1 Non-treatment related characteristics

\begin{tabular}{ll}
\hline Variable & Breast cancer survivors $(\boldsymbol{n}=\mathbf{3 0})$ \\
\hline Age $(\mathrm{y})$ & $50.5 \pm 5.6$ \\
Time since diagnosis (months) & $58 \pm 27$ \\
BMl $\left(\mathrm{kg} \mathrm{m}^{-2}\right)$ & $29.2 \pm 5.3$ \\
Body fat (\%) & $44.5 \pm 7.7$ \\
Overweight (BMI $>25 \mathrm{kgm}^{-2}$ ) & $25 / 30(83 \%)$ \\
Hypertension & $8 / 30(27 \%)$ \\
$\uparrow$ LDL & $20 / 30(66 \%)$ \\
Family history heart disease & $12 / 30(40 \%)$ \\
Sedentary lifestyle & $10 / 30(33 \%)$ \\
Smoker & $1 / 30(3 \%)$ \\
\hline
\end{tabular}

Note: $B M I$, body mass index; $L D L$, low density lipoprotein.

mouthpiece and headgear (Hans Rudolph; Shawnee, KS) before stepping on to the treadmill. Participants were encouraged to give a maximal effort during the test. The protocol involved a slow progression towards a static speed of $3.3 \mathrm{MPH}$ at the start of minute three, with an initial minute at 2.0 MPH and a second minute at 2.7 $\mathrm{MPH}$. At minute three the speed increased to $3.3 \mathrm{MPH}$ and a grade of $1 \%$. The grade increased $1 \%$ each minute thereafter. Participants continued walking on the treadmill at increasing incline until exhaustion, unless indications for terminating the maximal test were observed (ACSM 2009). Participants cooled-down at 1.5-2.0 MPH and a level grade for a period of two to five minutes. Expired air was analyzed for $\mathrm{O}_{2}$ and $\mathrm{CO}_{2}$. Heart rate, blood pressure, and rating of perceived exertion score were determined at the end of each two minute stage. Confirmation of a maximal effort was determined by meeting three out of four of the following criteria; 1) plateau in heart rate and oxygen uptake with increased workload, 2) respiratory exchange ratio $>1.1$, 3) rating of perceived exertion $>17$, and 4) heart rate $>$ $90 \%$ of age predicted maximal heart rate.

\section{Submaximal treadmill test}

Cardiorespiratory fitness submaximal endpoints on the treadmill were determined by the $\mathrm{VO}_{2}$ at anaerobic threshold and $85 \%$ age predicted maximum heart rate. Initial criteria for anaerobic threshold was established by using the respiratory exchange ratio $\geq 1.0$. Participants were allowed to continue for three consecutive 15 second averages at a respiratory exchange ratio $\geq 1.0$ to ensure anaerobic threshold was reached. Post-test confirmation of the anaerobic threshold was made upon visual assessment of 1) V-slope method, and 2) ventilatory equivalent technique to rule out hyperventilation. Submaximal $\mathrm{VO}_{2}$ at $85 \%$ age predicted maximum heart rate was assessed with the calculation [(220-age $) \times 0.85]$ and determination of the corresponding $\mathrm{VO}_{2}$ at that heart rate during maximal testing on the treadmill.

\section{Submaximal arc (Cybex 750AT) test}

After a brief familiarization trial followed by a short rest period, participants began the Arc submaximal test. Participants' weight was input into the console before starting the test. The manual mode was used for testing, with initial workload set at a resistance level of 15 . Participants were asked to maintain between $80-100$ strides per minute and a level grade was used throughout the test. Workload was adjusted to allow a steady increase in heart rate until subjects reached the anaerobic threshold endpoint. Increased workload was performed manually by a gradual increase in wattage while adjusting resistance in two minute stages. Resistance at each two minute stage varied between participants to allow a steady increase in heart rate, and was adjusted by a $5-10$ numerical increase on the Arc trainer console resistance key pad. The cardiorespiratory fitness submaximal endpoint on the Arc trainer was determined by the $\mathrm{VO}_{2}$ at anaerobic threshold. Criteria for anaerobic threshold was determined by the same method as the submaximal treadmill endpoint.

\section{Cardiorespiratory fitness $\left(\mathrm{VO}_{2 \max }\right)$}

Cardiorespiratory fitness was measured by the participants' $\mathrm{VO}_{2 \max }$ during the maximal treadmill test. Group mean $\mathrm{VO}_{2 \max }$ was compared to the normative $\mathrm{VO}_{2 \max }$ value for healthy age-matched women (ACSM 2009). We performed our maximal treadmill test with a similar Balke protocol that was used for determining normative $\mathrm{VO}_{2 \max }$ values.

\section{Left ventricular ejection fraction}

Resting LVEF was performed on all breast cancer survivors by either an ECHO or MUGA, depending on which cardiac function test the participant had prior to starting breast cancer treatment. As previously mentioned, all breast cancer survivor participants had an ECHO or MUGA test within 2 weeks of their cardiorespiratory test. Cardiac function (LVEF) was assessed by a cardiologist not associated with the study.

\section{Anthropometrics and body composition}

Body weight was assessed to the nearest $0.1 \mathrm{~kg}$, using an electronic scale (Health o meter, Boca Raton, Fl). A stadiometer was used to measure height without shoes to the nearest $0.1 \mathrm{~cm}$ (SECA). Body composition was measured by dual-energy $x$-ray absorptiometry in the total body scanning mode with a Lunar Prodigy DXA machine (Lunar Corp., Madison, WI). Body mass index was calculated as the weight in kilograms divided by the height in meters squared $\left(\mathrm{kg} \mathrm{m}^{-2}\right)$. 


\section{Other study measures}

Blood pressure and resting heart rate was measured at rest prior to exercise testing. Family history of heart disease, sedentary lifestyle, and smoking habits was assessed from the chart review, clinical interview, and self-report during initial screening for the study.

\section{Statistical analysis}

Pre-formatted data collection and case report forms were used for both clinical and laboratory data. Study data was entered into an Excel database by a trained data clerk not related to the study. Values and percentages were used to describe $\mathrm{VO}_{2 \max }$ in breast cancer survivors compared to age and gender matched normative values. Distribution normality and was verified using the Shapiro-Wilk test and variance homogeniety was confirmed from a Levene's test. After assumptions were met, an association between all submaximal $\mathrm{VO}_{2}$ endpoints and $\mathrm{VO}_{2 \max }$ was examined using Pearson correlation coefficients. Correlations were categorized as 0.26 to 0.49 is a low correlation, 0.50 to 0.69 is moderate, 0.70 to 0.89 is high, and 0.90 to 1.00 is very high as described by Munro et al. (Munro 1993). All analyses were two-tailed with alpha $=0.05$, and performed with SPSS statistical software (version 15.0; SPSS Inc, Chicago, IL).

\section{Results}

\section{Cohort characteristics}

Cohort characteristics and non-treatment CVD risk factors are listed in Table 1. Mean age was $50.5 \pm 5.6$ years, $29 / 30$ were caucasion, $1 / 30$ was Latino, and the group had an average elapsed time since diagnosis of 58 months. The most frequent non-treatment related risk factors were BMI $>25 \mathrm{~kg} / \mathrm{m}^{2}$ (83\%), elevated LDL (66\%), family history of heart disease (40\%), and sedentary lifestyle (33\%). Additionally, the group mean body fat $\%$ was $44.5 \pm 7.7 \%$. Treatment related risk factors are listed in Table 2. 28/30 (93\%) of the breast cancer survivors were postmenopausal at time of study, while $20 / 30(66 \%)$ of the women were treated with an aromatase inhibitor. A

Table 2 Treatment related characteristics

\begin{tabular}{ll}
\hline Variable & Breast cancer survivors $(\boldsymbol{n}=\mathbf{3 0})$ \\
\hline Current Aromatase Inhibitor & $14 / 30(47 \%)$ \\
Current Tamoxifen & $1 / 30(3 \%)$ \\
Past Aromatase Inhibitors & $4 / 30(13 \%)$ \\
Past Tamoxifen & $1 / 30(3 \%)$ \\
Post-menopausal & $28 / 30(93 \%)$ \\
Adriamycin/Epirubicin & $24 / 30(80 \%)$ \\
Herceptin & $8 / 30(27 \%)$ \\
Left chest radiation & $12 / 30(40 \%)$ \\
\hline
\end{tabular}

large majority $(80 \%)$ of the women received either doxorubicin or epirubicin, $27 \%$ were treated with trastuzamab, and $40 \%$ had left chest radiation. Also, the group mean LVEF at time of study was $60.5 \pm 5 \%$, which was significantly decreased $(\mathrm{p}=0.02)$ from $63.2 \pm 5.7 \%$ at time of diagnosis and prior to treatment.

\section{Cardiorespiratory fitness levels}

All cardiorespiratory fitness measures are listed in Table 3. 23/30 (77\%) of the breast cancer survivors with 2 or more CVD risk factors tested below the 20th percentile consistent with low cardiorespiratory fitness based on their age group and gender for $\mathrm{VO}_{2 \max }$ as established by the American College of Sports Medicine (ACSM 2009), despite a mean time since diagnosis of over 4 years and a normal group mean LVEF at time of study. All submaximal testing of $\mathrm{VO}_{2}$ endpoints showed a high correlation to the actual measured $\mathrm{VO}_{2 \max }$ when comparing within subject results (Figure 1), and the $\mathrm{VO}_{2}$ at $85 \%$ age predicted maximum heart rate on the treadmill showed the highest correlation with $\mathrm{VO}_{2 \max }$. Our analysis of cardiorespiratory fitness revealed that the breast cancer survivor group's mean $\mathrm{VO}_{2 \max }(25.4 \pm$ $5.3 \mathrm{~mL} \cdot \mathrm{kg}^{-1} \cdot \mathrm{min}^{-1}$ ) was similar to the 20th percentile threshold value $\left(25.1 \mathrm{mLkg}^{-1 .} \mathrm{min}^{-1}\right)$ for age and gender group matched normative values.

\section{Adverse events from testing}

All 30 maximal and 30 submaximal cardiorespiratory tests were completed without any adverse events. During maximal testing twenty-eight of thirty participants met

\section{Table 3 Cardiorespiratory outcomes}

\begin{tabular}{|c|c|}
\hline Variable & $\begin{array}{l}\text { Breast cancer survivors } \\
(n=30)\end{array}$ \\
\hline Maximal $\mathrm{VO}_{2}\left(\mathrm{~mL}^{\prime} \mathrm{kg}^{-1} \cdot \mathrm{min}^{-1}\right)$ & $25.4 \pm 5.3$ \\
\hline Maximal HR (bpm) & $169 \pm 12$ \\
\hline $\mathrm{VO}_{2}$ at AT on treadmill $\left(\mathrm{mL} \cdot \mathrm{kg}^{-1} \cdot \mathrm{min}^{-1}\right)$ & $20.5 \pm 4.3$ \\
\hline $\mathrm{VO}_{2}$ at $A T$ on $\operatorname{Arc}\left(\mathrm{mL}^{\mathrm{kgg}}{ }^{-1} \cdot \mathrm{min}^{-1}\right)$ & $19.0 \pm 4.4$ \\
\hline $\mathrm{VO}_{2}$ at $85 \%$ APMHR $\left(\mathrm{mL}^{\mathrm{kg}}{ }^{-1} \cdot \mathrm{min}^{-1}\right)$ & $19.9 \pm 3.0$ \\
\hline HR at AT on treadmill (bpm) & $148 \pm 13$ \\
\hline HR at AT on Arc (bpm) & $144 \pm 13$ \\
\hline HR at $85 \%$ APMHR (bpm) & $144 \pm 5$ \\
\hline Predicted Maximal HR (bpm) & $170 \pm 6$ \\
\hline Predicted $\mathrm{VO}_{2}-85 \%$ APMHR $\left(\mathrm{mL}^{\prime} \mathrm{kg}^{-1} \cdot \mathrm{min}^{-1}\right)$ & $24.5 \pm 7.2$ \\
\hline Predicted $\mathrm{VO}_{2 \max }\left(\mathrm{mL} \cdot \mathrm{kg}^{-1} \cdot \mathrm{min}^{-1}\right)$ & $34.5 \pm 8.8$ \\
\hline Resting HR (bpm) & $78 \pm 10$ \\
\hline Maximal $\mathrm{O}_{2}$ pulse (mL/beat) & $11.5 \pm 1.5$ \\
\hline Pre-Treatment LVEF & $63.2 \pm 5.7 \%$ \\
\hline Study LVEF & $60.5 \pm 5.0 \%$ \\
\hline
\end{tabular}

Note: $H R$, heart rate; $A T$, anaerobic threshold; $A P M H R$, age predicted maximal heart rate; $\angle V E F$, left ventricular ejection fractions. 


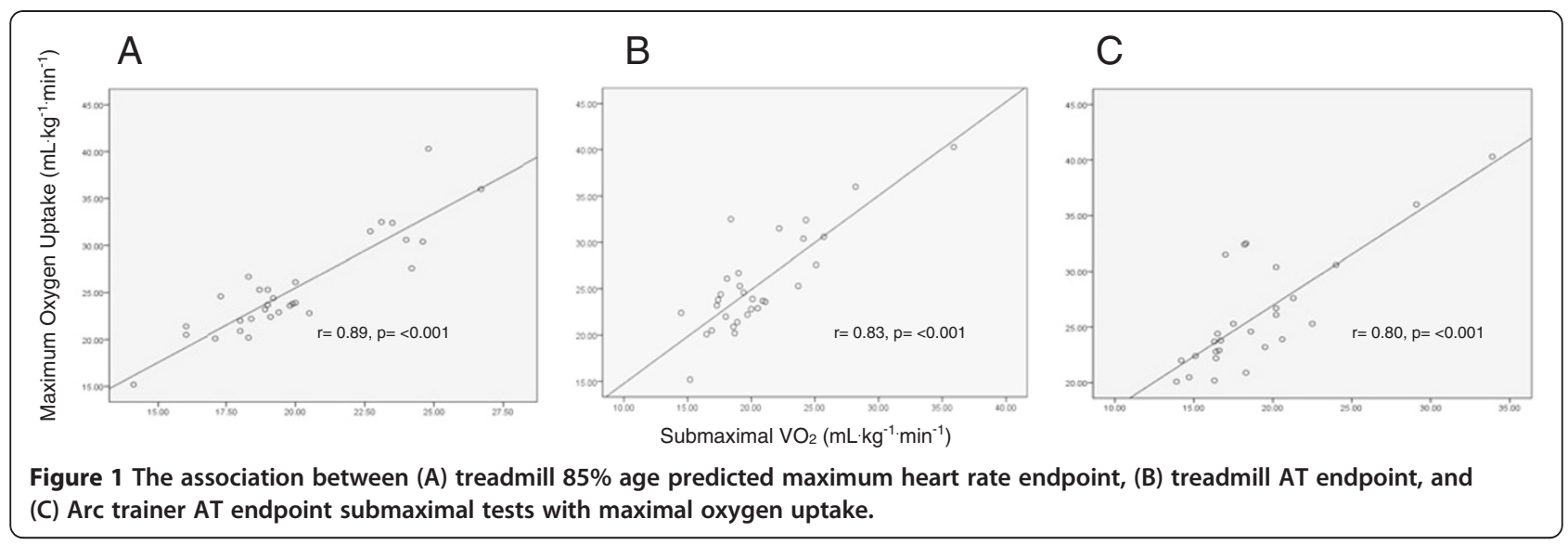

our established criteria for a maximal effort as explained previously. Two of the participants discontinued the test secondary to a claustrophobic sensation related to the mouthpiece, fatigue, and/or shortness of breath. Symptoms were resolved by discontinuing the test and having the participants rest while monitoring all posttest vitals. Even though two participants discontinued the maximal test prior to reaching maximal criteria, both participants achieved $>85 \%$ APMRH on the treadmill and have been included in the analyses.

\section{Discussion}

In our breast cancer survivors cohort, all participants had $\geq 2$ risk factors for CVD not related to treatment and $77 \%$ of this group had low cardiorespiratory fitness measured by $\mathrm{VO}_{2 \max }$ despite a normal LVEF at time of study. These results suggest that they are at a higher risk of breast cancer and cardiovasular mortality (Blair et al. 1996; Blair et al. 1995; Peel et al. 2009). Our results are similar to three earlier reports in breast cancer survivors, (one of which women had controlled hypertension), suggesting the majority of women with non-treatment related CVD risk factors have a lower $\mathrm{VO}_{2 \max }$ compared to healthy women even with a concomitant normal LVEF (Jones et al. 2012; Jones et al. 2007b; Tolentino et al. 2010). Our study differs from the earlier studies since we used a similar $\mathrm{VO}_{2 \max }$ protocol (Balke protocol) to the standardized treadmill test used for determining normative values (ACSM 2009) and we reported pre- and post-treatment LVEF.

Collectively, the double impact from the effects of lifestyle changes and breast cancer adjuvant treatment may increase the risk for late-onset CVD. Despite this knowledge, there is not a current stratification tool to accurately assess increased risk of CVD morbidity and mortality in breast cancer survivors. For example, subclinical cardiac dysfunction may go unnoticed until more overt symptoms occur and still remain undetected by a resting echocardiogram (ECHO) (Cardinale et al. 2004;
Civelli et al. 2006). However, exercise tests may be more sensitive than resting tests in identifying cardiac dysfunction in long-term survivors (Gottdiener et al. 1981; Klewer et al. 1992; Weesner et al. 1991). Therefore, exercise testing may serve as an important clinical tool for identifying breast cancer survivors who are asymptomatic, but at increased risk for the development of CVD. Furthermore, cardiorespiratory exercise testing can provide an objective evaluation of cardiorespiratory fitness, reducing the variability found in self-reported activity measures by $70-80 \%$ (Blair \& Church 2004).

Sub-maximal exercise testing with $\mathrm{VO}_{2}$ measured at the anaerobic threshold has shown good correlation with maximal exercise testing in individuals where a maximum test would be difficult because of disability or deconditioning including those with congestive heart failure, stroke, or undergoing bone marrow transplant (Carlson et al. 2006; Eng et al. 2004; Kemps et al. 2008). To our knowledge, this is the first study to investigate the association between maximal and submaximal cardiorespiratory fitness testing in breast cancer survivors. We found that submaximal $\mathrm{VO}_{2}$ endpoints were highly correlated with $\mathrm{VO}_{2 \max }$, indicating that submaximal testing can be a good measure of cardiorespiratory fitness in breast cancer survivors. Our study produced similar results when comparing submaximal $\mathrm{VO}_{2}$ at anaerobic threshold and $85 \%$ age predicted maximum heart rate to $\mathrm{VO}_{2 \max }$, suggesting that submaximal testing can be used as a surrogate for $\mathrm{VO}_{2 \max }$ testing in breast cancer survivors.

The submaximal $\mathrm{VO}_{2}$ at $85 \%$ age predicted maximum heart rate was used because this predetermined endpoint can be performed without expensive gas analysis equipment and can be more feasible than measuring anaerobic threshold when a large number of patients or subjects need to be tested. Submaximal $\mathrm{VO}_{2}$ endpoint at $85 \%$ age predicted maximum heart rate had a similar group mean heart rate and $\mathrm{VO}_{2}$ compared to the anaerobic threshold endpoint. This is important to note since 
anaerobic threshold is a helpful indicator for determining fitness level and for measuring the effect of exercise training (Casaburi 1994; Casaburi et al. 1991). Overall, the submaximal $\mathrm{VO}_{2}$ endpoint at $85 \%$ age predicted maximum heart rate showed the highest correlation to actual measured $\mathrm{VO}_{2 \max }$, as seen in Figure 1. Our findings suggest using the speed and grade at the $85 \%$ age predicted maximum heart rate endpoint during a submaximal test for predicting $\mathrm{VO}_{2}$ and objectively measuring cardiorespiratory fitness for breast cancer survivors, especially when repeated measures are required for assessing improvement after an exercise intervention. Our results support the use of a submaximal cardiorespiratory test as an objective measure of fitness that can be used for breast cancer survivors. Furthermore, this study suggests that a submaximal cardiorespiratory test using a modified Balke protocol with an endpoint set at $85 \%$ age predicted maximum heart rate can be performed when the equipment and personnel needed to conduct directly measured oxygen uptake via indirect calorimetry are not available. A validation study to design an accurate predictive model for extrapolating $\mathrm{VO}_{2 \max }$ from a submaximal testing end point at the $85 \%$ age predicted maximum heart rate endpoint.

We are unaware of previous studies that have used an Arc trainer with decreased load bearing force as an exercise testing modality to examine cardiorespiratory fitness in breast cancer survivors, many of whom have agerelated or aromatase inhibitor induced arthalgia and may prefer alternate forms of testing. Turner et al. showed that $\mathrm{VO}_{2 \max }$ and time to attain $\mathrm{VO}_{2 \max }$ were similar when comparing results between testing modalities including an Arc trainer and a treadmill in healthy adults (Turner et al. 2010). During our study three participants were unable to complete testing on the Arc trainer due to their inability to coordinate the movement between the lower and upper limbs simultaneously. Also, the Arc trainer produced significantly lower $\mathrm{VO}_{2}$ and heart rate at anaerobic threshold versus the treadmill submaximal test (Table 3). Overall, we suggest that the benefit of using an Arc trainer does not outweigh difficulty with performing the required coordinated movements and lack of standard testing protocol. Also, It is important to note that there were no breast cancer survivors in our study that could not complete testing on the treadmill due to joint or muscle pain.

This is the first study to investigate the association between the gold standard for cardiorespiratory fitness $\left(\mathrm{VO}_{2 \max }\right)$ to submaximal $\mathrm{VO}_{2}$ tests in breast cancer survivors who had been treated with adjuvant therapy. The findings from this study indicate that breast cancer survivors with $\geq 2$ CVD risk factors had low cardiorespiratory fitness and submaximal testing on the treadmill is a feasible, objective measure of fitness that can be used in breast cancer survivors. Limitations of this study include the small sample size $(n=30)$, lack of racial diversity, and lack of non-cancer controls.

Future research should focus on using a high-risk breast cancer control group with $\geq 2 \mathrm{CVD}$ risk factors to compare maximal and sub-maximal $\mathrm{VO}_{2}$ measures. In addition, novel approaches should be made to improve cardiorespiratory fitness during sustainable exercise interventions for breast cancer survivors.

\section{Ethical standards}

All work described within this manuscript complies with United States and institutional regulations for the protection of human subjects.

\section{Competing interests}

The authors declare that they have no competing interests.

\section{Authors' contributions}

DB conceived the study, developed the study methods, and drafted the manuscript. JK helped with the development of the study methods, was in charge of participant recruitment, managed the IRB approval process, and edited the manuscript. PK helped develop the study methods, was in charge of reviewing testing protocols and Clinical Translational Science Unit procedures, and edited the manuscript. CP was in charge of reviewing procedures for assessing left ventricular ejection fraction and edited the manuscript. CF conceptualized and guided the most appropriate breast cancer participant selection and edited the manuscript. All authors read and approved the final manuscript.

Authors' information

Co-authors: Patricia Kluding, Charles Porter, Carol Fabian, Jennifer Klemp.

\section{Acknowledgements}

The authors thank Colby Greer for his help in developing the Arc trainer protocol for this study. In addition Catie Knight for the support with recruitment and Sonya Aversman for coordinating scheduling of all tests. Lastly, we wish to thank all the participants for their time and efforts.

\section{Sources of funding}

This trial was funded by the NIH BIRCWH K12 Program (HD052027) from NIH BIRCWH K12 (Klemp) and Back in the Swing (Klemp). Also, this work was supported in part by Frontiers: The Heartland Institute for Clinical and

Translational Research (University of Kansas Medical Center CTSA;

UL1RR033179). No funding is declared by the remaining authors.

Received: 12 February 2013 Accepted: 18 February 2013

Published: 25 February 2013

\section{References}

ACS (2012) Cancer facts and figures 2012. http://www.cancer.org/Research/ CancerFactsFigures/CancerFactsFigures/cancer-facts-figures-2011. Accessed May 112012

ACSM (2009) ACSM's guidelines for exercise testing and prescription, 8th edn Lippincott, Williams \& Wilkins, Baltimore

Andrykowski MA, Curran SL, Lightner R (1998) Off-treatment fatigue in breast cancer survivors: a controlled comparison. J Behav Med 21(1):1-18

Balke BWR (1959) An experimental study of physical fitness in Air Force personnel. US Armed Forces Med J 10:675-688

Bernardi M, Guerra E, Di Giacinto B, Di Cesare A, Castellano V, Bhambhani Y (2010) Field evaluation of paralympic athletes in selected sports: implications for training. Med Sci Sports Exerc 42(6):1200-1208. doi:10.1249/ MSS.0b013e3181c67d82

Blair SN, Church TS (2004) The fitness, obesity, and health equation: is physical activity the common denominator? JAMA 292(10):1232-1234. doi:10.1001/ jama.292.10.1232 [doi] 292/10/1232 [pii] 
Blair SN, Kohl HW 3rd, Paffenbarger RS Jr, Clark DG, Cooper KH, Gibbons LW (1989) Physical fitness and all-cause mortality. A prospective study of healthy men and women. JAMA 262(17):2395-2401

Blair SN, Kohl HW 3rd, Barlow CE, Paffenbarger RS Jr, Gibbons LW, Macera CA (1995) Changes in physical fitness and all-cause mortality. A prospective study of healthy and unhealthy men. JAMA 273(14):1093-1098

Blair SN, Kampert JB, Kohl HW 3rd, Barlow CE, Macera CA, Paffenbarger RS Jr, Gibbons LW (1996) Influences of cardiorespiratory fitness and other precursors on cardiovascular disease and all-cause mortality in men and women. JAMA 276(3):205-210

Cardinale D, Sandri MT, Colombo A, Colombo N, Boeri M, Lamantia G, Civelli M, Peccatori F, Martinelli G, Fiorentini C, Cipolla CM (2004) Prognostic value of troponin I in cardiac risk stratification of cancer patients undergoing highdose chemotherapy. Circulation 22:2749-2754. doi:10.1161/01. CIR.0000130926.51766.CC [doi] 01.CIR.0000130926.51766.CC [pii]

Carlson LE, Smith D, Russell J, Fibich C, Whittaker T (2006) Individualized exercise program for the treatment of severe fatigue in patients after allogeneic hematopoietic stem-cell transplant: a pilot study. Bone Marrow Transplant 37 (10):945-954. doi:10.1038/sj.bmt.1705343 [doi] 1705343 [pii]

Casaburi R (1994) Physiologic responses to training. Clin Chest Med 15(2):215-227

Casaburi R, Patessio A, Ioli F, Zanaboni S, Donner CF, Wasserman K (1991) Reductions in exercise lactic acidosis and ventilation as a result of exercise training in patients with obstructive lung disease. Am Rev Respir Dis 143 (1):9-18

Civelli M, Cardinale D, Martinoni A, Lamantia G, Colombo N, Colombo A, Gandini S, Martinelli G, Fiorentini C, Cipolla CM (2006) Early reduction in left ventricular contractile reserve detected by dobutamine stress echo predicts high-dose chemotherapy-induced cardiac toxicity. Int J Cardiol 111(1):120-126. doi:10.1016/j.jijcard.2005.07.029 S0167-5273(05)01069-7 [pii]

Eloranta S, Lambert PC, Andersson TM, Czene K, Hall P, Bjorkholm M, Dickman PW (2012) Partitioning of excess mortality in population-based cancer patient survival studies using flexible parametric survival models. BMC Med Res Methodol 12(1):86. doi:10.1186/1471-2288-12-86 [doi]1471-2288-12-86 [pii]

Eng JJ, Dawson AS, Chu KS (2004) Submaximal exercise in persons with stroke: testretest reliability and concurrent validity with maximal oxygen consumption. Arch Phys Med Rehabil 85(1):113-118. [doi] S0003999303004362 [pii]

Ewer MS, Swain SM, Cardinale D, Fadol A, Suter TM (2011) Cardiac dysfunction after cancer treatment. Tex Heart Inst J 38(3):248-252

Gitt AK, Wasserman K, Kilkowski C, Kleemann T, Kilkowski A, Bangert M, Schneider S, Schwarz A, Senges J (2002) Exercise anaerobic threshold and ventilatory efficiency identify heart failure patients for high risk of early death. Circulation 106(24):3079-3084

Gottdiener JS, Mathisen DJ, Borer JS, Bonow RO, Myers CE, Barr LH, Schwartz DE, Bacharach SL, Green MV, Rosenberg SA (1981) Doxorubicin cardiotoxicity: assessment of late left ventricular dysfunction by radionuclide cineangiography. Ann Intern Med 94(4 pt 1):430-435

Jones LW, Haykowsky MJ, Swartz JJ, Douglas PS, Mackey JR (2007a) Early breast cancer therapy and cardiovascular injury. J Am Coll Cardiol 50(15):1435-1441

Jones LW, Haykowsky M, Peddle CJ, Joy AA, Pituskin EN, Tkachuk LM, Courneya KS, Slamon DJ, Mackey JR (2007b) Cardiovascular risk profile of patients with HER2/ neu-positive breast cancer treated with anthracycline-taxane-containing adjuvant chemotherapy and/or trastuzumab. Cancer Epidemiol Biomarkers Prev 16(5):1026-1031. doi:10.1158/1055-9965.EPI-06-0870 [doi] 16/5/1026 [pii]

Jones LW, Courneya KS, Mackey JR, Muss HB, Pituskin EN, Scott JM, Hornsby WE, Coan AD, Herndon JE 2nd, Douglas PS, Haykowsky M (2012) Cardiopulmonary function and age-related decline across the breast cancer survivorship continuum. J Clin Oncol 30(20):2530-2537. doi:10.1200/ JCO.2011.39.9014 [doi] JCO.2011.39.9014 [pii]

Kemps HM, Schep G, de Vries WR, Schmikli SL, Zonderland ML, Thijssen EJ, Wijn PF, Doevendans PA (2008) Predicting effects of exercise training in patients with heart failure secondary to ischemic or idiopathic dilated cardiomyopathy. Am J Cardiol 102(8):1073-1078. doi:10.1016/j. amjcard.2008.05.054 [doi] S0002-9149(08)01019-9 [pii]

Klewer SE, Goldberg SJ, Donnerstein RL, Berg RA, Hutter JJ Jr (1992) Dobutamine stress echocardiography: a sensitive indicator of diminished myocardial function in asymptomatic doxorubicin-treated long-term survivors of childhood cancer. J Am Coll Cardiol 19(2):394-401. [doi] 0735-1097(92)90497-B [pii]

Lu TW, Chien HL, Chen HL (2007) Joint loading in the lower extremities during elliptical exercise. Med Sci Sports Exerc 39(9):1651-1658. doi:10.1249/ mss.0b013e3180dc9970 [doi] 00005768-200709000-00028 [pii]
McNeely ML, Campbell KL, Rowe BH, Klassen TP, Mackey JR, Courneya KS (2006) Effects of exercise on breast cancer patients and survivors: a systematic review and meta-analysis. CMAJ 175(1):34-41. doi:10.1503/cmaj.051073 [doi] 175/1/34 [pii]

Munro B (1993) Correlations. Statistical methods for health care research. JP Lippincott, Philadelphia

NCEP (2002) Third report of the National Cholesterol Education Program (NCEP) expert panel on detection, evaluation, and treatment of high blood cholesterol in adults (adult treatment panel III) final report. Circulation 106 (25):3143-3421

Peel JB, Sui X, Adams SA, Hebert JR, Hardin JW, Blair SN (2009) A prospective study of cardiorespiratory fitness and breast cancer mortality. Med Sci Sports Exerc 41(4):742-748. doi:10.1249/MSS.0b013e31818edac7

Tolentino GP, Battaglini CL, Araujo SS, Otano AS, Conde DM, Evans ES, de Oliveira RJ (2010) Cardiorespiratory fitness and quality-of-life analysis posttreatment in breast cancer survivors. J Psychosoc Oncol 28(4):381-398. doi:10.1080/ 07347332.2010 .484831 [doi] 924051220 [pii]

Turner MJ, Williams AB, Williford AL, Cordova ML (2010) A comparison of physiologic and physical discomfort responses between exercise modalities. J Strength Cond Res 24(3):796-803. doi:10.1519/JSC.0b013e3181cc2472

Wasserman K, Hansen J, Sue D et al (1999) Principles of exercise testing and interpretation, 3rd edn. Lippincott Williams \& Wilkins, Philadelphia

Weesner KM, Bledsoe M, Chauvenet A, Wofford M (1991) Exercise echocardiography in the detection of anthracycline cardiotoxicity. Cancer 68(2):435-438

doi:10.1186/2193-1801-2-68

Cite this article as: Burnett et al:: Cardiorespiratory fitness in breast cancer survivors. SpringerPlus 2013 2:68.

\section{Submit your manuscript to a SpringerOpen ${ }^{\odot}$ journal and benefit from:}

- Convenient online submission

- Rigorous peer review

- Immediate publication on acceptance

- Open access: articles freely available online

- High visibility within the field

- Retaining the copyright to your article

Submit your next manuscript at $>$ springeropen.com 